\title{
Clinical Significance of Upregulation of mir-196a-5p in Gastric Cancer and Enriched KEGG Pathway Analysis of Target Genes
}

\author{
Hai-Long Li ${ }^{1,2,3 \&}$, Shou-Pin Xie ${ }^{4 \&}$, Ya-Li Yang ${ }^{1}$, Ying-Xia Cheng ${ }^{2}$, Ying Zhang ${ }^{5}$, \\ Jing Wang ${ }^{1}$, Yong Wang ${ }^{1}$, Da-Long Liu ${ }^{4}$, Zhao-Feng Chen ${ }^{3}$, Yong-Ning Zhou ${ }^{3}$, \\ Hong-Yan $\mathrm{Wu}^{4 *}$
}

\begin{abstract}
Background: miRNAs are relatively recently discovered cancer biomarkers which have important implications for cancer early diagnosis, treatment and estimation of prognosis. Here we focussed on expression of mir-196a-5p in gastric cancer tissues and cell lines so as to analyse its significance for clinicopathologic characteristics and generate enriched KEGG pathways clustered by target genes for exploring its potential roles as a biomarker in gastric cancer. Materials and Methods: The expression of mir-196a-5p in poorly, moderate and well differentiated gastric cancer cell lines compared with GES-1 was detected by RT-qPCR, and the expression of mir-196a-5p in gastric cancer tissues comparing with adjacent non cancer tissues of 58 cases were also assessed by RTqPCR. Subsequently, an analysis of clinical significance of mir-196a-5p in gastric cancer and enriched KEGG pathways was executed based on the miRWalk prediction database combined with bioinformatics tools DAVID 6.7 and Mirfocus 3.0. Results: RT-qPCR showed that mir-196a-5p was up-regulated in 6 poorly and moderate differentiated gastric cancer cell lines SGC-7901, MKN-45, MKN-28, MGC-803, BGC-823, HGC-27 compared with GES-1, but down-regulated in the highly differentiated gastric cancer cell line AGS. Clinical data indicated mir-196a-5p to beup-regulated in gastric cancer tissues $(47 / 58)$. Overexpression of mir-196a-5p was associated with more extensive degree of lymph node metastasis and clinical stage $(P<0.05$; 22 test). Enriched KEGG pathway analyses of predicted and validated targets in miRWalk combined with DAVID 6.7 and Mirfocus 3.0 showed that the targeted genes regulated by mir-196a-5p were involved in malignancy associated biology. Conclusions: Overexpression of mir-196a-5p is associated with lymph node metastasis and clinical stage, and enriched KEGG pathway analyses showed that targeted genes regulated by mir-196a-5p may contribute to tumorgenesis, suggesting roles as an oncogenic miRNA biomarker in gastric cancer.
\end{abstract}

Keywords: Gastric cancer - mir-196a-5p - oncogenic gene - KEGG pathway

Asian Pac J Cancer Prev, 16 (5), 1781-1787

\section{Introduction}

Gastric cancer (GC) is the fifth most common cancer but the third leading cause of cancer death (Fock, 2014), and the common characteristics of GC is high incidence rates, poorly early diagnosis rates and high mortality rates. Epidemiology data indicated that the current trend of GC is severe worldwide, particular in Asia (Katanoda et al., 2013; Liu et al., 2013; Koessler et al., 2014). The major reason of which is associated with lack of specificity of symptoms for early diagnosis and treatment. For example, GC is diagnosed at an early stage in less than $10 \%$ of cases in Europe (Saika and Sobue, 2013). The funding and identification of new molecular biomarkers made it easier for GC diagnosis, treatment and clinical outcome prognosis, and microRNAs (miRNAs) were one of new discovered cancer molecular biomarkers relevant to cancer including GC. MiRNAs are 20-to-25 mer noncoding RNAs which incompletely bind to the 3' untranslated regions (UTR) of multiple target mRNAs, enhancing their degradation and inhibiting their translation. MiRNAs participate in multiple carcinogenesis processes, such as cell differentiation, cell cycle, metastasis, progression and apoptosis, and even microRNA polymorphism is closed correlated with cancer (Hao et al., 2013; Du et al., 2014a). Aberrantly expressed miRNAs act as tumor suppressor

${ }^{I}$ Department of Clinical Testing Teaching and Research, School of Medical Technology, Gansu Traditional Chinese Medical University, ${ }^{2}$ Key Laboratory of Traditional Chinese Herbs and Prescription Innovation and Transformation in Gansu Province, Gansu Traditional Chinese Medical University, ${ }^{3}$ Division of Gastroenterology and Hepatology, First Hospital of Lanzhou University, ${ }^{4}$ First People's Hospital of Lanzhou City, ${ }^{5}$ Department of Dermatology, The First Hospital of Chinese People's Liberation Army, Lanzhou, China ${ }^{\circledR}$ Equal contributors*For correspondence: wu.hy@126.com 
genes or oncogenic genes in cancer tumorigenesis as well as in gastric cancer(Shenouda and Alahari, 2009; Bartels and Tsongalis, 2010; Cortes-Sempere and Ibanez de Caceres, 2011). Mir-196a is a newly reported miRNA biomarkers relevant to multiple cancers, such as breast cancer (Lee et al., 2014), lung cancer (Liu et al., 2012), cervical cancer (Gocze et al., 2013), renal cancer (Du et al., 2014a), head and neck cancer (Christensen et al., 2010), hepatocellular cancer (Hao et al., 2013), pancreatic cancer (Slater et al., 2014), colorectal cancer (Zhan et al., 2011; Du et al., 2014b) and early gastric cancer (Zheng et al., 2014).

In previous study, mir-196a-5p was discovered over expressed in gastric cancer cell lines and gastric cancer tissues (Sun et al., 2012; Tsai et al., 2014). But the clinical significance of mir-196a-5p in cancers of previous reports was different, probably, which may be caused by different representatives of different samples. So expanded study will clarify its clinical significance and understand its roles in gastric cancer, and will make it useful for GC cancer molecular biomarkers discovery. In present study, we decided to validate the expression of mir-196a-5p in seven gastric cancer cell lines comparing with GES-1 cell line, and detect the expression of mir-196a-5p in gastric cancer tissues comparing with adjacent non-cancerous tissues of 58 cases comes from a high gastric cancer incidental rate area. In addition, bioinformatics analysis of its target genes and enriched KEGG pathways will be execute to understand its roles in cancer tumorigenesis, particularly in the case of gastric cancer.

\section{Materials and Methods}

\section{Cell lines}

Seven gastric cancer cell lines, AGS, SGC-7901, MKN-45, MKN-28, MGC-803, BGC-823, HGC-27 and GES-1 were purchased from the Type Culture Collection of the Chinese Academy of Sciences (Shanghai, China) and Cancer Institute and Hospital, Chinese Academy of Medical Sciences (CAMS) (Beijing, China). All the gastric cancer cell lines were maintained in DMEM supplemented with $10 \%$ heat-inactivated fetal bovine serum in a humidified cell incubator having an atmosphere of 5\% $\mathrm{CO} 2$ at $37^{\circ} \mathrm{C}$. Exponentially growing cells were used for experiments. MiRNA PCR primers were purchased from GeneCopoeia $^{\mathrm{TM}}$ Inc.

\section{Clinical samples}

58 gastric cancer samples were obtained during surgery and used after obtaining informed consent. All patients underwent curative resection of the primary tumor at WuWei city tumor Hospital from the year of 2010 to 2012 (WuWei, China, a high incidental rate area with gastric cancer (Li et al., 2004; ZHANG et al., 2009). All patients had a clear histological diagnosis of gastric cancer, based on the clinicopathologic criteria. All data, including age, sex, histological grade, depth, lymph node metastasis, Local invasion, Depth of tumor invasion, lymph node metastasis, lymphatic invasion, Venous invasion, borrmann type and clinical stage were obtained from clinical and pathologic records. No patients received neoadjuvant chemotherapy or radiotherapy before surgery and adjuvant radiotherapy after surgery. Resected cancerous tissues ( $\mathrm{T}$ ) and paired noncancerous tissues $(\mathrm{N})$ were immediately cut and stored in, frozen in liquid nitrogen, and kept at- $80^{\circ} \mathrm{C}$ until RNA extraction.

Written informed consent was obtained from each patient for his or her participation in the study. The study protocol conformed to the ethical guidelines of the 1975 Declaration of Helsinki as reflected in a priori approval by the ethics committee of First Hospital of Lanzhou University.

\section{Total RNA isolation and quality analysis}

Total RNA of gastric cancer cell lines and frozen tissues of gastric cancer were extracted using RNeasy mini kit (QIAGEN, Hilgen, Germany) according to the manufacturer's instructions. Concentrations and purity of the RNA samples were assayed by electrophoresis and spectrophotometric methods.

\section{MiRNA quantification by $q R T-P C R$}

MiRNA quantification by real-time qRT-PCR. SYBR green qRT-PCR assay was used for miRNA quantification. In brief, $40 \mathrm{ng}$ of total RNA containing miRNA was polyadenylated by poly(A) polymerase and was reversely transcripted to cDNA using miScript Reverse Transcription kit according to the manufacturer's instructions (GeneCopoeia ${ }^{\mathrm{TM}}$, Rockville, USA). miScript SYBR Green PCR kit was used and miscript Universal primer was provided by the manufacturer (GeneCopoeia ${ }^{\mathrm{TM}}$, Rockville, USA), qRT-PCR was performed in BIORAD CFX96 Real-time PCR system. Each reaction was performed in a final volume of $10 \mu \mathrm{l}$ containing $2 \mu \mathrm{l}$ of cDNA, $0.5 \mathrm{mM}$ of each primer and 1X SYBR Green PCR Master mix (GeneCopoeia ${ }^{\mathrm{TM}}$, Rockville, USA). The amplification program was: denaturation at $95^{\circ} \mathrm{C}$ for 10 min, followed by 40 cycles of $95^{\circ} \mathrm{C}$ for $10 \mathrm{sec}, 60^{\circ} \mathrm{C}$ for $30 \mathrm{sec}$ and $72^{\circ} \mathrm{C}$ for $30 \mathrm{sec}$, in which fluorescence was acquired. At the end of the PCR cycles, melting curve analyses were performed as well as electrophoresis of the products on $2.5 \%$ agarose gels in order to validate the specific generation of the expected PCR product. Each sample was run in triplicates for analysis. The expression levels of miRNAs were normalized to RNU6B. Relative gene expression was calculated as 2-(CTmiRNACTRNU6B RNA).

MiRNA Targeted Gene Prediction and KEGG Pathway Analyses by miRWalk and DAVID 6.7

We utilized a miRNA target gene prediction database miRWalk (Dweep et al., 2011) to select predicted and validated targets, and to analysis enriched KEGG pathways by bioinformatics tool DAVID 6.7. The miRWalk prediction database integrated 10 bioinformatics Target Prediction Tools: DIANA-mT, miRanda, miRDB, miRWalk, RNAhybrid, PICTAR4, PICTAR5, PITA, RNA22 and TargetScan. Enriched KEGG pathway analyses of mir-196a-5p targeted genes were performed by bioinformatics tool DAVID 6.7. Prediction Databases Support Number were at least 5, P value of Fisher Test were $\mathrm{P}<0.05$. 
MiRNA Targeted Gene Prediction and KEGG Pathway Analyses by mirfocus 3.0

We utilized a miRNA target gene prediction database mirfocus 3.0 (http://mirfocus.org/index.php) to select validated targets of mir-196a-5p to analysis its enriched KEGG pathways and to annotate the molecular function of the miRNA targeted genes. The mirfocus 3.0 integrated 5 bioinformatical Target Prediction Tools: MiRanda, MirTarget2, PicTar, microT and TargetScanS, and the experimental validated Target Tools include miRecords, miR2Disease, TarBase and miRTarBase. Enriched KEGG pathway of mir-196a-5p targeted genes also were performed by mirfocus 3.0. Prediction Databases Support Number was 3, $\mathrm{P}$ value of Fisher Test were $\mathrm{P}<0.05$.

\section{Statistical analysis}

Student's unpaired t-test was used to compare values of samples of 7 gastric cancer cell lines and samples of GES-1 gastric cell line. Differences between groups were estimated using the $\mathrm{X}^{2}$ test. A probability level of 0.05 was chosen for statistical significance, and all statistical analyses were performed using the SPSS 17.0 software (SPSS Inc., Chicago, IL, USA).

\section{Results}

Expression of mir-196a-5p in gastric cancer cell lines

The expression of mir-196a-5p in gastric cancer cell lines comparing with GES-1 was detected by qRTPCR method. Results showed that mir-196a-5p were up-regulated in 6 gastric cancer cell lines gastric cancer cell lines SGC-7901, MKN-45, MKN-28, MGC-803, BGC-823, HGC-27 compared with GES-1 cell line, but down-regulated in gastric cancer cell line AGS (Figure 1).

Expression of mir-196a-5p in gastric cancer tissues comparing with adjacent non tumor tissues

After detection the expression of mir-196a-5p in gastric cancer cell lines, the levels of mir-196a-5p in 58 cancerous and corresponding non-cancerous tissues were also detected by qRT-PCR method. Results showed that the numbers of mir-196a-5p in high-expression group $(\mathrm{T} /$ $\mathrm{N}>2)$ and low-expression group $(\mathrm{T} / \mathrm{N}<0.5)$ amounts to 47 and 11 respectively, according to the median cancer $(\mathrm{T}) /$ noncancerous $(\mathrm{N})$ tissue ratio of mir-196a-5p expression (Figure 2, Table 1).

Table 1. mir-196a-5p Level and Clinocopathologic Factors in Patients with Gastric Cancer

\begin{tabular}{|c|c|c|c|c|}
\hline$\overline{\text { Characteristic }}$ & $\begin{array}{c}\text { mir-196a-5p } \\
\text { High expression } \\
(\mathrm{n}=47) \\
\text { Number }(\%)\end{array}$ & $\begin{array}{c}\text { mir-196a-5p } \\
\text { Low expression } \\
(\mathrm{n}=11) \\
\text { Number }(\%)\end{array}$ & significance & $\mathrm{P}$ value \\
\hline$\overline{\text { Age }}$ & & & 0.113 & 0.737 \\
\hline$<59$ years & $29(61.7 .0 \%)$ & $8(72.7 \%)$ & & \\
\hline$\geq 59$ years & $18(38.3 \%)$ & $3(27.3 \%)$ & & \\
\hline Gender & & & 0.416 & 0.519 \\
\hline Males & $29(61.7 .0 \%)$ & $5(45.4 .0 \%)$ & & \\
\hline Females & $18(38.3 \%)$ & $6(54.6 \%)$ & & \\
\hline Degree of tumour cell differentiation & & & 0.000 & 1.000 \\
\hline Moderate-to-well differentiated & $20(42.6 \%)$ & $4(36.4 \%)$ & & \\
\hline Poorly differentiated & $30(57.4 \%)$ & $7(63.6 \%)$ & & \\
\hline TNM stage & & & 4.958 & $0.026 \mathrm{a}$ \\
\hline $\mathrm{I}+\mathrm{II}$ & $9(20.0 \%)$ & $6(76.0 \%)$ & & \\
\hline $\mathrm{III}+\mathrm{IV}$ & $38(80.0 \%)$ & $5(24.0 \%)$ & & \\
\hline Tumor size & & & 1.283 & 0.257 \\
\hline$\leq 5 \mathrm{~cm}$ & $21(20.0 \%)$ & $7(28.0 \%)$ & & \\
\hline$>5 \mathrm{~cm}$ & $26(80.0 \%)$ & $4(72.0 \%)$ & & \\
\hline Lymph node metastasis & & & 4.273 & $0.039 \mathrm{a}$ \\
\hline Positive & $35(74.4 \%)$ & $4(36.4 \%)$ & & \\
\hline Negative & $12(25.6 \%)$ & $7(63.6 \%)$ & & \\
\hline Lymphatic invasion & & & 0.024 & 0.878 \\
\hline Positive & $42(89.4 \%)$ & $10(90.9 \%)$ & & \\
\hline Negative & $5(10.6 \%)$ & $1(9.1 \%)$ & & \\
\hline Venous invasion & & & 0.004 & 0.951 \\
\hline Positive & $4(8.5 \%)$ & $1(9.1 \%)$ & & \\
\hline Negative & $43(91.5 \%)$ & $10(90.9 \%)$ & & \\
\hline Depth of tumor invasiona & & & 1.010 & 0.315 \\
\hline mucosa, submucosa, muscularis propria, subserosa & $22(46.8 \%)$ & $7(63.6 \%)$ & & \\
\hline penetration of serosa, adjacent strucures & $25(53.2 \%)$ & $4(36.4 \%)$ & & \\
\hline Borrmann type & & & 0.009 & 0.925 \\
\hline $\mathrm{I}+\mathrm{II}$ & $29(61.7 .0 \%)$ & $6(54.5 \%)$ & & \\
\hline $\mathrm{III}+\mathrm{IV}$ & $18(38.3 \%)$ & $5(45.5 \%)$ & & \\
\hline
\end{tabular}

The expression of mir-196a-5p in all cases showed different levels between gastric cancer tissues and adjacent non-cancerous tissues. a $\mathrm{P}<0.05$, lymph node metastasis; TNM stage 
Table 2. Enriched KEGG Pathway Clustered by Predicted Targets of mir-196a-5p and Corresponding Target Genes. 'Fisher-P-value' Stands for the Enrichment $p$-value of the Pathway ID using the Fisher's Exact Test. Enrichment Score' Stands for the Enrichment score value of the Pathway ID Qqualing '-log10 (P value)'

\begin{tabular}{|c|c|c|c|}
\hline KEGG PATHWAY & Fold Enrichment & Fisher Exact & Predicted target genes \\
\hline Prostate cancer & 3.4 & $6.40 \mathrm{E}-06$ & $\begin{array}{l}\text { BCL2,CREB3L1,CDKN1A,CDKN1B,FGFR1,FOXO1, } \\
\text { HSP90AA1,IKBKB,IGF1,MAPK1,NRAS, } \\
\text { NFKB1,NFKBIA,PDGFA,PDGFRA,TCF7,AKT1 }\end{array}$ \\
\hline Chronic myeloid leukemia & 3.1 & $2.20 \mathrm{E}-04$ & $\begin{array}{l}\text { CBL,SMAD4,CDK6,CDKN1A,CDKN1B,IKBKB,MA } \\
\text { PK1,NRAS,NFKB1,NFKBIA,PTPN11,TGFBR2,AKT1 }\end{array}$ \\
\hline Acute myeloid leukemia & 3.1 & $1.20 \mathrm{E}-03$ & $\begin{array}{l}\text { CEBPA,FLT3,IKBKB,MAPK1,NRAS,NFKB1,PML,ST } \\
\text { AT3,TCF7,AKT1 }\end{array}$ \\
\hline Glioma & 2.8 & $2.40 \mathrm{E}-03$ & $\begin{array}{l}\text { CAMK2G,CALM1,CALM3,CDK6,CDKN1A,IGF1,M } \\
\text { APK1,NRAS,PDGFA,PDGFRA,AKT1 }\end{array}$ \\
\hline Small cell lung cancer & 2.5 & $2.30 \mathrm{E}-03$ & $\begin{array}{l}\text { BCL2,XIAP,CDK6,CDKN1B,CDKN2B,IKBKB,ITGA } \\
\text { V,LAMA4,NFKB1,NFKBIA,RARB,AKT1 }\end{array}$ \\
\hline Melanogenesis & 2.5 & $1.10 \mathrm{E}-03$ & $\begin{array}{l}\text { ADCY1,ADCY6,ADCY9,CREB3L1,CAMK2G,CALM1, } \\
\text { CALM3,EDNRB,MAPK1,NRAS,PLCB2,PRKX,TCF7, } \\
\text { TYRP1,WNT2B }\end{array}$ \\
\hline Pancreatic cancer & 2.5 & $6.40 \mathrm{E}-03$ & $\begin{array}{l}\text { CBL,SMAD4,CDK6,CDKN1A,CDKN1B,IKBKB, } \\
\text { MAPK1,NRAS,NFKB1,NFKBIA,PTPN11,TGFBR2, } \\
\text { AKT1,VEGFA }\end{array}$ \\
\hline TGF-beta signaling pathway & 2.5 & $3.10 \mathrm{E}-03$ & $\begin{array}{l}\text { SMAD4,SMAD6,ACVR2A,ACVR2B,BMP4,BMPR2, } \\
\text { CHRD,CDKN2B,INHBC,MAPK1,ROCK1,TGFBR2 }\end{array}$ \\
\hline mTOR signaling pathway & 2.4 & $2.50 \mathrm{E}-02$ & RICTOR,IGF1,MAPK1,RPS6KA3,TSC1,AKT1,VEGFA \\
\hline Chemokine signaling pathway & 2.3 & $1.00 \mathrm{E}-04$ & $\begin{array}{l}\text { RAP1A,WASL,ADCY1,ADCY6,ADCY9,XCL1,CCL17, } \\
\text { CCL22,CCL23,CCR10,CXCL10,CXCL12,GNB3,IK, } \\
\text { KB,MAPK1,NRAS,NFKB1,NFKBIA,PLCB2,PRKX, } \\
\text { STAT2,STAT3 }\end{array}$ \\
\hline Melanoma & 2.3 & $1.70 \mathrm{E}-02$ & $\begin{array}{l}\text { CDK6,CDKN1A,FGFR1,IGF1,MAPK1,NRAS,PDGFA, } \\
\text { PDGFRA,AKT1 }\end{array}$ \\
\hline Pathways in cancer & 2.1 & 4.10E-06 & $\begin{array}{l}\text { BCL2,BAX,CEBPA,CBL,FAS,GLI3,SMAD4,XIAP, } \\
\text { BMP4,CDK6,CDKN1A,CDKN1B,CDKN2B, } \\
\text { FGFR1,FLT3,FOXO1,HSP90AA1,RALBP1,IKBKB, } \\
\text { IGF1,ITGAV,LAMA4,MAPK1,NRAS, } \\
\text { NFKB1,NFKBIA,PDGFA,PDGFRA,PML,RET,RARB, } \\
\text { STAT3,SHH,TCF7,TGFBR2,AKT1,ETS1,VEGFA, } \\
\text { WNT2B }\end{array}$ \\
\hline p53 signaling pathway & 2.1 & $3.50 \mathrm{E}-02$ & $\begin{array}{l}\text { BAX,FAS,CCND2,CDK6,CDKN1A,IGF1,SERPINE1, } \\
\text { ZMAT3 }\end{array}$ \\
\hline Renal cell carcinoma & 2 & 4.10E-02 & $\begin{array}{l}\text { RAP1A,MAPK1,NRAS,PAK2,PTPN11,AKT1, } \\
\text { ETS1,VEGFA }\end{array}$ \\
\hline
\end{tabular}

Table 3. Enriched KEGG Pathway Clustered by Validated Targets of mir-196a-5p and Corresponding Target Genes. 'Fisher-P-value' Stands for the Enrichment $p$-value of the Pathway ID using the Fisher's Exact Test. Enrichment Score' Stands for the Enrichment Score value of the Pathway ID Equaling '-log10 (P value) '.

\begin{tabular}{lcll}
\hline KEGG PATHWAY & Fold Enrichment & Fisher Exact & Validated target genes \\
\hline Acute myeloid leukemia & 8.9 & $2.10 \mathrm{E}-04$ & CEBPA,FLT3,IKBKB,NFKB1,AKT1 \\
Apoptosis & 6 & $1.40 \mathrm{E}-03$ & BAX DFFA IKBKB NFKB1 AKT1 \\
Renal cell carcinoma & 5.9 & $4.40 \mathrm{E}-03$ & AKT1, ETS1, VEGFA, RAP1A \\
Prostate cancer & 5.8 & $1.50 \mathrm{E}-03$ & AKT1, IGF1R, CDKN1A, NFKB1, IKBKB \\
Pancreatic cancer & 5.8 & $4.80 \mathrm{E}-03$ & AKT1, VEGFA, NFKB1, IKBKB \\
Chronic myeloid leukemia & 5.5 & $5.60 \mathrm{E}-03$ & AKT1, CDKN1A, NFKB1, IKBKB \\
Toll-like receptor signaling pathway & 5.1 & $2.70 \mathrm{E}-03$ & AKT1, IFNA1, NFKB1, TLR4, IKBKB \\
Small cell lung cancer & 4.9 & $8.30 \mathrm{E}-03$ & AKT1, ITGAV, NFKB1, IKBKB \\
Cell cycle & 4.2 & $6.70 \mathrm{E}-03$ & CDKN1A, CCND2, ESPL1, MCM2, SMC3 \\
Pathways in cancer & 4.1 & $8.30 \mathrm{E}-06$ & BMP4, CEBPA, FLT3, NFKB1, SHH, AKT1, IGF1R, \\
& & CDKN1A, ETS1, ITGAV, BAX, VEGFA, IKBKB \\
\hline
\end{tabular}

Clinical significances of mir-196a-5p in gastric cancer tissues comparing with adjacent non tumor tissues

All clinicopathologic factors were analyzed in relation to mir-196a-5p level, The mir-196a-5p high-expression group showed more lymph node metastasis and clinical stage than the high-expression group ( $p<0.05 ; \mathrm{X}^{2}$ test). However, no significant differences were observed among age, gender, extensive degree of tumor cell differentiation, tumor size, depth of tumor invasion, venous invasion, borrmann type, and lymphatic invasion (Table 1). 
Clinical Significance of mir-196a-5p in Gastric Cancer and Enriched KEGG Pathway Analysis of its Target Genes

Table 4. Enriched KEGG Pathway Clustered by Predicted and Validated Targets of mir-196a-5p and Corresponding Target Genes. Genes enrichment were Performed using the Fisher's exact test. Enrichment score' Stands for the Enrichment Score value of the Pathway ID equaling '-log10 (P value)

\begin{tabular}{lccl}
\hline KEGG PATHWAY & Fold Enrichment & Predicted target genes & Validated target genes \\
\hline MicroRNAs in cancer & 3.44 & HMGA2, NRAS,PDGFRA & CASP3,CCND1,CCND2,CCNE2, \\
& & & CDKN1B,HMOX1,IGF2BP,IKBKB, \\
NOTCH2, PDCD4 & CCND2,CCNT2,CDKN1B,EWSR1, \\
Transcriptional misregulation in cancer & 2.76 & ERG, HMGA2, PBX1, PBX3 & FOXO1,HOXA9,IGF1R, \\
Prostate cancer & 2.66 & NRAS, PDGFRA & CCND1,CCNE2,CDKN1B,FOXO1, \\
Glioma & 2.11 & CALM1,CALM3,PDGFRA & IGF1R,IKBKB \\
PI3K-Akt signaling pathway & 1.68 & COL1A1, COL1A2, COL24A1, & CCND1,CCND2,CCNE2,CDKN1B, \\
& & OSMR,COL3A1, NRAS, PDGFRA & IGF1R,IKBKB,ITGAV, \\
& 1.36 & & BUB1,CCND1,CCND2,CCNE2, \\
Cell cycle & & & CDKN1B,ESPL1,SMC3 \\
\end{tabular}

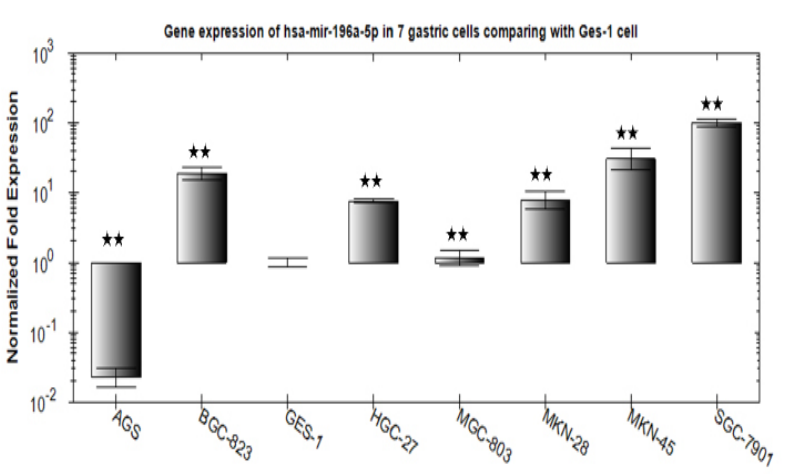

Figure 1. RT-qPCR Experiment Validation Showed that mir-196a-5p were up-regulated in 6 Gastric Cancer Cell Lines SGC-7901, MKN-45, MKN-28, MGC-803, BGC-823, HGC-27 Compared with GES-1 Cell line, but Down-regulated in Gastric Cancer Cell Line AGS. Relative gene expression was calculated as $2^{-\Delta \Delta C T}$ method, and each sample was analyzed in triplicate. there were statistically significant difference between 7 gastric cancer cell lines and GES- 1 cell line $(p<0.05$, indicated as $\star \star$

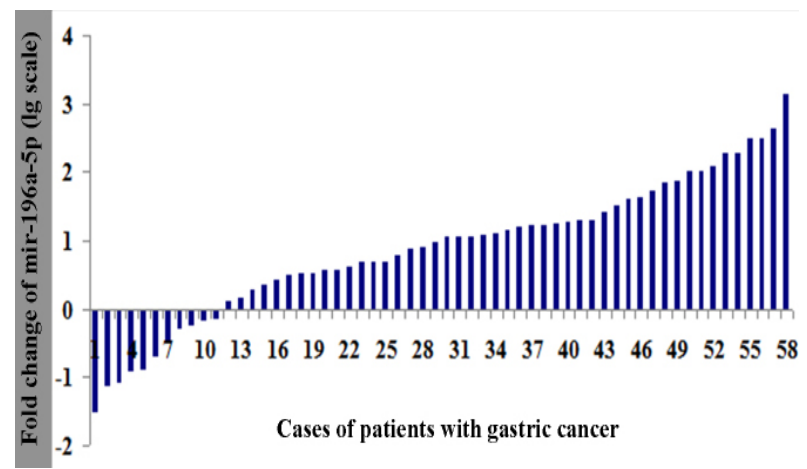

Figure 2. Expression of mir-196a-5p in Gastric Cancer Tissue Comparing with Adjacent Non Tumor Tissues of 58 Gastric Cancer Samples

KEGG pathway analyses of mir-196a-5p targeted genes by miRWalk and DAVID 6.7

In order to investigate the possible regulation mechanisms of mir-196a-5p in the process of gastric cancer, we utilized an online bioinformatics database miRWalk to select plausible targets and validated targets of this miRNA and to analysis Enriched KEGG pathways by DAVID 6.7 .

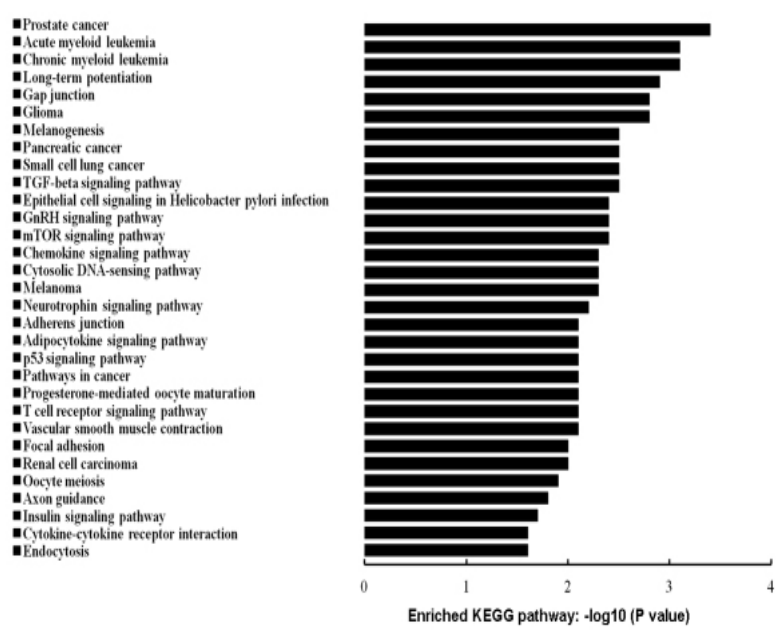

Figure 3. The 31 Enriched KEGG Pathways from Predicted Target Genes of mir-196a-5p which were Searched in MiRWalk Database and Analysed by DAVID 6.7

Based on predicted targets of miRWalk, a total of 1081 target genes were searched as the target genes of mir-196a-5p. Enriched KEGG pathway analyses showed that the targeted genes which were regulated by mir196a-5p were involved 31 pathways, and 13 of which were centralized in cancer associated terms, which were as follows: prostate cancer, chronic myeloid leukemia, acute myeloid leukemia, glioma, small cell lung cancer, melanogenesis, pancreatic cancer, TGF-beta signaling pathway, mTOR signaling pathway, chemokine signaling pathway, Melanoma, Pathways in cancer and p53 signaling pathway (Table 2, Figure 3).

Based on validated targets of miRWalk, a total of 209 target genes were searched as the target genes of mir-196a-5p. Enriched KEGG pathway analyses showed that the targeted genes which were regulated by mir196a-5p were involved 14 pathways, and 10 of which were centralized in cancer associated terms, which were as follows: acute myeloid leukemia, apoptosis, renal cell carcinoma, prostate cancer, pancreatic cancer, chronic myeloid leukemia, toll-like receptor signaling pathway, small cell lung cancer, cell cycle and pathways in cancer (Table 3, Figure 4). 


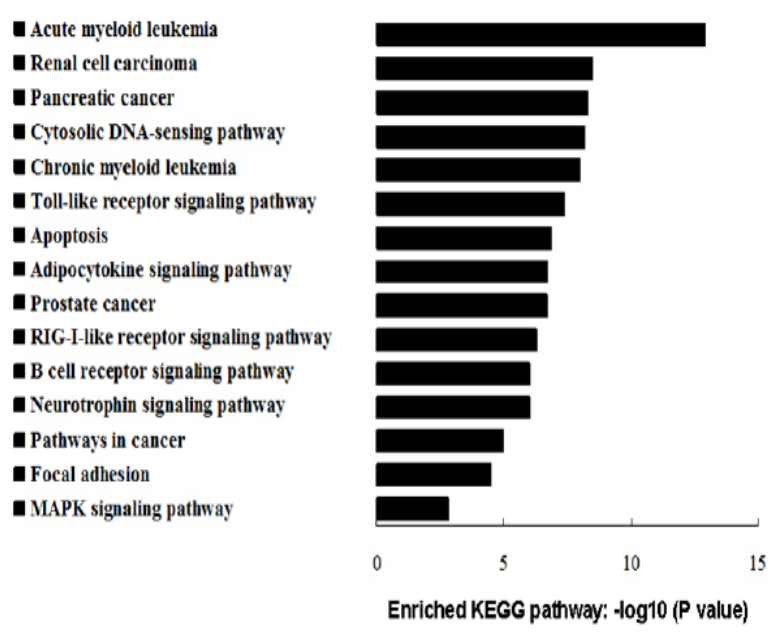

Figure 4. The 14 Enriched KEGG Pathways from Validated Target Genes of mir-196a-5p which were Searched in miRWalk Database and analysed by DAVID 6.7
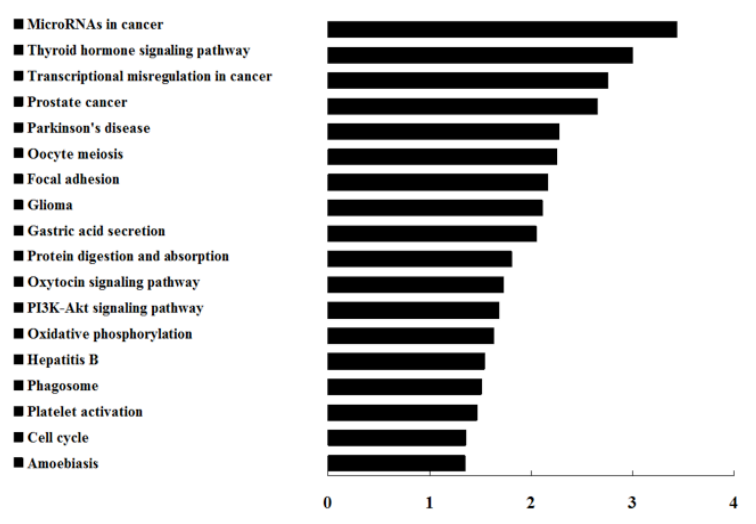

Figure 5. The 18 Enriched KEGG Pathways from Predicted and Validated Target Genes of mir-196a-5p which were Searched and Analysed in miRfocus 3.0 Database

KEGG pathway analyses of mir-196a-5p targeted genes by Mirfocus 3.0

In order to investigate the possible regulation mechanisms of mir-196a-5p in the process of gastric cancer, we utilized a bioinformatical database mirfocus 3.0 to select plausible targets of this miRNA. A total of 348 target genes including predicted and validated genes were choosed as the target genes of mir-196a-5p.

Results of enrichment KEGG pathway indicated that the targeted genes which were regulated by mir196a-5p were involved 18 pathways, and 6 of which were centralized in cancer associated terms, which were as follows: MicroRNAs in cancer, transcriptional misregulation in cancer, prostate cancer, glioma, cell cycle and PI3K-Akt signaling pathway ((Table 4, Figure 5).

\section{Discussion}

Aberrantly expressed miRNAs are unique cancer biomarkers which will bring benefit to early diagnosis, treatment guidelines and prognosis estimation of cancer as well as mRNA and protein biomarkers. In recent years, more and more miRNAs were found up-regulated or down-regulated in gastric cancer, so that they were potential biomarkers which will supplement classic cancer biomarkers in clinical applications, such as miR-21(Guo et al., 2013; Ma et al., 2013). But an ideal biomarker not only must be associated with cancer clinical significance, but also functional experiments of over expression or silence should prove it having great impact on cancer behavior. Mir-196a-5p was newly discovered promising gastric cancer biomarker, more studies should be carried out to fully understand its multiple roles and functions and to clarify any previous inconsistent clinical significance. This study employed 58 gastric cancer cases comes from WuWei city tumor Hospital from the year of 2010 to 2012 which belong to WuWei, Gan su, China, an area is famous for its high incidental rate with gastric cancer. So the clinical data is typical for clinical significance study, and is qualified for biomarker evaluation.

In this study, RT-qPCR experiment showed that mir196a-5p were up-regulated in 6 gastric cancer cell lines SGC-7901, MKN-45, MKN-28, MGC-803, BGC-823, HGC-27 compared with GES-1 cell line, but downregulated in gastric cancer cell line AGS. Among these seven gastric cancer cell lines, MGC-803 and HGC-27 were undifferentiated carcinoma cells, SGC-7901, MKN$45, \mathrm{MKN}-28$ and BGC-823 were poorly and moderate differentiated carcinoma cells, only AGS was highly differentiated carcinoma cell. So mir-196a-5p may be responsible for the differentiation of gastric cancer cell.

Clinical data analysis indicated that the numbers of mir-196a-5p in high-expression group $(\mathrm{T} / \mathrm{N}>2)$ and low-expression group $(\mathrm{T} / \mathrm{N}<0.5)$ amounts to 47 and 11 respectively. The mir-196a-5p high-expression group showed more extensive degree of lymph node metastasis and clinical stage than the high-expression group $(\mathrm{P}<$ 0.05 ; $\mathrm{x} 2$ test). However, no significant differences were observed among age, gender, depth of tumor invasion, venous invasion, tumor cell differentiation, tumor size, Borrmann type, and lymphatic invasion.

Previous study discovered that overexpression of miR196a was significantly associated with tumor progression and poorer 5-year survival outcomes, and overexpression of miR-196a enhanced GC cell migration and invasion (Tsai et al., 2014). In another study (Sun et al., 2012), higher expression of miR-196a in gastric cancer tissues was reported associated with tumor size, higher clinical stage, and shorter overall survival, and downregulation of miR-196a expression significantly suppressed the cellcycle progression, proliferation, and colony formation of gastric cancer cells in vitro, and ectopic miR-196a expression significantly enhanced the development of tumors in nude mice. Result of our study is partly consistent with these studies.

Bioinformatics analysis of plausible targets and validated targets of miRNA and following analysis of KEGG pathway clustered by target genes is a promising way to give insights on potential biomarkers and key events involved in cancer tumorigenesis and relevant pathways. In recent years, this kind of combination analysis of any identified miRNA, its corresponding target 
genes, enriched KEGG pathways, and even miRNAmRNA network were performed in cancer studies (Satoh, 2012; Liu et al., 2014), particularly in cancer biomarker discoveries (Romero-Cordoba et al., 2012; Beta et al., 2013). In this study, based on miRWalk database combined with DAVID 6.7 tool and mirfocus 3.0, bioinformatics prediction and Enriched KEGG pathways analysis of miR-196a-5p indicated that both predicted target genes and validated target genes of miR-196a were clustered in cancer associated KEGG pathways.

In conclusion, our study fully reinforced the expressions of mir-196a-5p in gastric cancer lines and gastric cancer tissues were up regulated. These results, combined with bioinformatics analysis of Enriched KEGG pathways clustered by target genes provide ways to identify mir196a-5p as new biomarkers in gastric cancer, and new light should be shed on its clinical significances of gastric cancer diagnosis and prognosis.

\section{Acknowledgements}

This study was supported by the Foundation of the fundamental scientific research funds for colleges and universities in Gansu Province (no. [2014]63-15).

\section{References}

Bartels CL, Tsongalis GJ (2010). MicroRNAs: novel biomarkers for human cancer. Ann Biol Clin (Paris), 68, 263-72 (in French).

Beta M, Venkatesan N, Vasudevan M, et al (2013). Identification and insilico analysis of retinoblastoma serum microRNA profile and gene targets towards prediction of novel serum biomarkers. Bioinform Biol Insights, 7, 21-34.

Christensen BC, Avissar-Whiting M, Ouellet LG, et al (2010). Mature microRNA sequence polymorphism in MIR196A2 is associated with risk and prognosis of head and neck cancer. Clin Cancer Res, 16, 3713-20.

Cortes-Sempere M, Ibanez de Caceres I (2011). microRNAs as novel epigenetic biomarkers for human cancer. Clin Transl Oncol, 13, 357-62.

Du M, Lu D, Wang Q, et al (2014a). Genetic variations in microRNAs and the risk and survival of renal cell cancer. Carcinogenesis, 35, 1629-35.

Du W, Ma XL, Zhao C, et al (2014b). Associations of single nucleotide polymorphisms in miR-146a, miR-196a, miR-149 and miR-499 with colorectal cancer susceptibility. Asian Pac J Cancer Prev, 15, 1047-55.

Dweep H, Sticht C, Pandey P, et al (2011). miRWalk--database: prediction of possible miRNA binding sites by "walking" the genes of three genomes. J Biomed Inform, 44, 839-47.

Fock KM (2014). Review article: the epidemiology and prevention of gastric cancer. Aliment Pharmacol Ther, 40, 250-60.

Gocze K, Gombos K, Juhasz K, et al (2013). Unique microRNA expression profiles in cervical cancer. Anticancer Res, 33, 2561-7.

Guo B, Li J, Liu L, et al (2013). Dysregulation of miRNAs and their potential as biomarkers for the diagnosis of gastric cancer. Biomed Rep, 1, 907-12.

Hao YX, Wang JP, Zhao LF (2013). Associations between three common microRNA polymorphisms and hepatocellular carcinoma risk in Chinese. Asian Pac J Cancer Prev, 14, 6601-4.
Katanoda K, Matsuda T, Matsuda A, et al (2013). An updated report of the trends in cancer incidence and mortality in Japan. Jpn J Clin Oncol, 43, 492-507.

Koessler T, Roth A, Cacheux W (2014). [Early gastric cancer: epidemiology, diagnostic and management]. Rev Med Suisse, 10, 1118-22.

Lee SJ, Seo JW, Chae YS, et al (2014). Genetic polymorphism of miR-196a as a prognostic biomarker for early breast cancer. Anticancer Res, 34, 2943-9.

Li Y-m, Shi B, Li X, et al (2004). Study of the characteristics of gastric carcinoma in Wuwei City of Gansu province. Chinese JGeneral Surgery, 13, 667-9.

Liu B, Li J, Cairns MJ (2014). Identifying miRNAs, targets and functions. Brief Bioinform, 15, 1-19.

Liu SZ, Wang B, Zhang F, et al (2013). Incidence, survival and prevalence of esophageal and gastric cancer in Linzhou city from 2003 to 2009. Asian Pac J Cancer Prev, 14, 6031-4.

Liu XH, Lu KH, Wang KM, et al (2012). MicroRNA-196a promotes non-small cell lung cancer cell proliferation and invasion through targeting HOXA5. BMC Cancer, 12, 348.

Ma GJ, Gu RM, Zhu M, et al (2013). Plasma post-operative miR-21 expression in the prognosis of gastric cancers. Asian Pac J Cancer Prev, 14, 7551-4.

Romero-Cordoba S, Rodriguez-Cuevas S, Rebollar-Vega R, et al (2012). Identification and pathway analysis of microRNAs with no previous involvement in breast cancer. PLoS One, 7, 31904 .

Saika K, Sobue T (2013). Cancer statistics in the world. Gan To Kagaku Ryoho, 40, 2475-80.

Satoh J (2012). Molecular network analysis of human microRNA targetome: from cancers to Alzheimer's disease. BioData $\operatorname{Min}, \mathbf{5}, 17$.

Shenouda SK, Alahari SK (2009). MicroRNA function in cancer: oncogene or a tumor suppressor? Cancer Metastasis Rev, 28, 369-78.

Slater EP, Strauch K, Rospleszcz S, et al (2014). MicroRNA-196a and $-196 \mathrm{~b}$ as potential biomarkers for the early detection of familial pancreatic cancer. Transl Oncol, 7, 464-71.

Sun M, Liu XH, Li JH, et al (2012). MiR-196a is upregulated in gastric cancer and promotes cell proliferation by downregulating p27(kip1). Mol Cancer Ther, 11, 842-52.

Tsai MM, Wang CS, Tsai CY, et al (2014). MicroRNA-196a/$196 \mathrm{~b}$ promote cell metastasis via negative regulation of radixin in human gastric cancer. Cancer Lett, 351, 222-31.

Zhan JF, Chen LH, Chen ZX, et al (2011). A functional variant in microRNA-196a2 is associated with susceptibility of colorectal cancer in a Chinese population. Arch Med Res, 42, 144-8.

ZHANG Z-y, WU Z-q, WANG H-j (2009). An analysis of gastric cancer detection and epidemicity in high incidental area with gastric cancer, Wuwei city, Gansu province. China Cancer, 10, 14

Zheng G, Xiong Y, Xu W, et al (2014). A two-microRNA signature as a potential biomarker for early gastric cancer. Oncol Lett, 7, 679-84. 